\title{
Effect of Surgical Treatment on Lipid Peroxidation Parameters and Antioxidant Status in the Serum of Patients with Peripheral Arterial Disease
}

\author{
Krzysztof Wojciech Strzyżewski, ${ }^{1}$ Maria Pioruńska-Stolzmann, ${ }^{2}$ Wacław Majewski, ${ }^{3}$ \\ Magdalena Kasprzak, ${ }^{1}$ and Wojciech Strzyżewski ${ }^{4}$ \\ ${ }^{1}$ Department of General Chemistry and Clinical Biochemistry, Poznan University of Medical Sciences, \\ ul. Grunwaldzka 6, 60-780 Poznań, Poland \\ ${ }^{2}$ Department of Clinical Biochemistry and Laboratory Medicine, Poznan University of Medical Sciences, \\ ul. Grunwaldzka 6, 60-780 Poznań, Poland \\ ${ }^{3}$ Department of General and Vascular Surgery, Poznan University of Medical Sciences, ul. Długa 1/2, 61-848 Poznań, Poland \\ ${ }^{4}$ Department of Orthopedics and Traumatology, Poznan University of Medical Sciences, ul. 28 Czerwca 1956 r. 135/147, \\ 61-545 Poznań, Poland
}

Correspondence should be addressed to Krzysztof Wojciech Strzyżewski; k.strzyzewski@ump.edu.pl

Received 27 June 2013; Revised 16 October 2013; Accepted 17 October 2013

Academic Editor: Luisella Bocchio-Chiavetto

Copyright (C) 2013 Krzysztof Wojciech Strzyżewski et al. This is an open access article distributed under the Creative Commons Attribution License, which permits unrestricted use, distribution, and reproduction in any medium, provided the original work is properly cited.

\begin{abstract}
The various risk factors for peripheral arterial disease (PAD) are almost identical to those for atherosclerosis and include abnormal levels of lipids or lipoproteins. Lipid peroxidation parameters and total antioxidant capacity in the serum of male patients with PAD before surgery as well as 3-5 days and 7-10 days after surgery were measured. We also compared these parameters with those in a group of patients receiving simvastatin therapy. Concentrations of lipid hydroperoxides (LOOHs) and malondialdehyde, the total antioxidant capacity (assessed by ferric reducing antioxidant power assay), concentration of thiol (-SH) groups, and ceruloplasmin activity were determined spectrophotometrically in PAD patients treated surgically (Group I) or pharmacologically (Group II). The patients before surgical treatment had significantly higher concentrations of malondialdehyde but lower ceruloplasmin activity than those observed in Group II, treated with simvastatin. No significant differences before surgery in ferric reducing antioxidant power or thiol concentrations were found between the two groups. However, in Group I, both ferric reducing antioxidant power and thiol group concentrations decreased 3-5 days postoperatively, and ceruloplasmin activity increased 7-10 days after surgical treatment. The presented results demonstrate diverse oxidative stress responses to surgical treatment and confirm the beneficial effects of statin therapy in PAD.
\end{abstract}

\section{Introduction}

Peripheral arterial disease (PAD) commonly results from progressive narrowing of arteries in the lower extremities due to atherosclerosis [1]. It has become evident that PAD is an important predictor of substantial coronary and cerebral vascular risk $[2,3]$. The most common symptomatic manifestation of mild to moderate atherosclerotic PAD is intermittent claudication [4], observed in $~ 5 \%$ of individuals older than 60 years $[5,6]$. Several studies, using the anklebrachial index, have revealed that the presence of PAD, even when being asymptomatic and in patients with no history of other cardiovascular diseases, is a marker of greatly increased cardiovascular morbidity and mortality $[7,8]$. Oxidative stress, through the extra generation of reactive oxygen species (ROS), is also involved in the development and progression of PAD [9].

Moreover, risk factors associated with PAD [10], similar to those for coronary heart disease [11], include high blood concentrations of total cholesterol and LDL-cholesterol. Inhibitors of 3-hydroxy-3-methylglutaryl coenzyme A (HMG-CoA) reductase, called statins, are well-known 
lipid-lowering drugs. Statins not only reduce lipid levels, but they also have important pleiotropic effects, improving endothelial function, reducing oxidative stress, affecting the lipid metabolism enzyme activities, and so forth [12-14]. Three main reasons have been suggested for initiating statin therapy for patients with PAD of the lower limbs [15]: (1) prevention of coronary heart disease; (2) treatment of lower extremity peripheral arterial disease; and (3) perioperative treatment of patients with peripheral arterial disease [16].

Although several trials have shown that treatment with statins is associated with a reduction in coronary heart disease events [17], such as stroke [18, 19] and mortality [20-23], most of these trials did not specifically address the treatment of patients with PAD. The aim of the present study, therefore, was to evaluate lipid peroxidation parameters and total antioxidant capacity in the serum of patients with PAD before surgery as well as 3-5 days and 7-10 days after surgery. We also compared these parameters with those in a group of patients receiving simvastatin therapy.

\section{Material and Methods}

2.1. Experimental Subjects. The study group consisted of PAD patients, treated either surgically (Group I, $n=35$ males, aged $61 \pm 8$ years) or pharmacologically (Group II, $n=18$ males, aged $62 \pm 7$ years) in the Department of General and Vascular Surgery at the Poznan University of Medical Sciences. Patients included in Group I were admitted for surgical treatment (implantation of an aortobifemoral bypass graft). They were diagnosed by measurement of the ankle brachial index (ABI) and arteriography of the lower limbs, and were clinically stable without any accompanying diseases. Subjects with diabetes were excluded. The patients treated surgically had shown critical ischemia (ABI less than 0.90, rest pain, ulcer or necrosis of the lower limbs, and ankle pressure $\leq$ $50 \mathrm{mmHg}$ ). Blood samples were collected without anticoagulant, in the fasting state, before surgery as well as 3-5 days and 7-10 days after surgical treatment. Group II had been receiving statin therapy, with $20 \mathrm{mg}$ of simvastatin daily, for at least 3 months. The study protocol was approved by the Ethics Committee of Poznan University of Medical Sciences, and the subjects were fully informed and gave their written consent.

2.2. Reagents and Apparatus. All the reagents were of analytical grade of purity (Sigma-Aldrich, St. Louis, MO, USA). Spectrophotometric measurements were carried out on SP8001 UV/Visible Spectrophotometer (Metertech Inc., Taiwan).

2.3. Procedures. The subjects' lipid profile (total cholesterol, HDL cholesterol, LDL cholesterol, and triglycerides), glucose, uric acid, apolipoproteins (Apo A-I, Apo B) and fibrinogen concentrations in serum were measured by standard laboratory procedures, using the Roche Cobas 6000 analyzer system, Dade Behring BN II system, and the Sysmex CA500 Series system. The total antioxidant potential (capacity) of serum was determined by ferric reducing antioxidant power (FRAP) assay according to the Benzie and Strain method [24], based on the reduction of ferric ion Fe(III) to ferrous ion $\mathrm{Fe}(\mathrm{II})$ at a low $\mathrm{pH}$. This method measures the change in absorbance at $593 \mathrm{~nm}$ owing to the formation of a blue-colored $\mathrm{Fe}(\mathrm{II})$-tripyridyltriazine complex from the colorless oxidized Fe(III) form, by the action of electrondonating antioxidants.

Lipid peroxidation was measured by estimation of both lipid hydroperoxides (LOOHs) and malondialdehyde (MDA) concentrations by the Sodergren method [25] and Ohkawa et al. method [26], respectively. The concentration of thiol ($\mathrm{SH}$ ) groups was determined according to the Hu method [27]. The oxidase activity of ceruloplasminin serum was determined spectrophotometrically according to the Schosinsky method [28]. Ceruloplasmin, in the presence of $o$-dianisidine hydrochloride in an acidic solution ( $\mathrm{pH}$ 5.0), forms a stable yellow-brownish product. Absorbance was measured at $540 \mathrm{~nm}$ and ceruloplasmin activity was expressed in U/L ( $\mu \mathrm{mol}$ of oxidized substrate in $1 \mathrm{~mL}$ of serum in $1 \mathrm{~min}$ ).

2.4. Statistical Analysis. The means and standard errors of the mean were calculated. Statistical analysis was performed using Instant GraphPad software. The paired Student's $t$-test (to compare the values before and after surgery), the twotailed unpaired $t$-test (to compare Group I and Group II), and the $F$-test were used for statistical analysis of the data.

The mean changes $(\Delta)$ in lipid peroxidation parameters and antioxidant capacity, that is, 3-5 days $\left(\Delta_{1}\right)$ and 7-10 days $\left(\Delta_{2}\right)$ after surgery, in relation to the values obtained before surgery, were calculated. Pearson's correlation coefficients $r_{1}$ and $r_{2}$ were computed for the results found before surgery and the changes observed during treatment $\left(\Delta_{1}, \Delta_{2}\right)$. Statistical significance was accepted at the $P<0.05$ level.

\section{Results}

As shown in Table 1, generally, the clinical parameters of the two groups of patients included in this study did not differ significantly. However, the concentrations of total cholesterol and LDL cholesterol were found to be significantly higher in Group I before surgery than those in Group II on simvastatin therapy.

Group I patients before surgery had enhanced oxidative stress, as demonstrated by elevated serum levels of MDA, in comparison to those treated pharmacologically. We did not observe any significant decrease in $\mathrm{LOOH}$ and MDA concentrations in Group I patients after surgery (Figures 1 and 2).

The total antioxidant capacity (expressed as FRAP) and -SH group concentrations in the serum of patients on statin therapy and in those before surgery were similar (Figures 3 and 4). However, a decrease was observed in FRAP level 3-5 days after surgery, and 7-10 days after surgery, it was significantly lower in comparison to the values before surgery and on simvastatin therapy. Moreover, thiol group concentrations 3-5 days postoperatively were lower than those before surgery and 7-10 days after surgery and were lower than those in patients treated with simvastatin (Figure 4).

A significant difference in the oxidase activity of ceruloplasmin between Group II and Group I before surgery was observed. Its oxidase activity in serum was higher in Group II 
TABLE 1: Clinical characteristics of Group I patients (before surgery) and Group II patients (treated pharmacologically with simvastatin).

\begin{tabular}{lccc}
\hline Parameter & Group I before surgery & Group II treated with simvastatin & $P$ value \\
\hline Total cholesterol [mmol/L] & $5.9 \pm 0.9$ & $5.1 \pm 1.1$ & $<0.05$ \\
HDL cholesterol [mmol/L] & $1.2 \pm 0.3$ & $1.3 \pm 0.4$ & $\mathrm{~ns}$ \\
LDL cholesterol [mmol/L] & $4.2 \pm 1.5$ & $3.0 \pm 1.1$ & $<0.05$ \\
Triacylglycerols [mmol/L] & $1.8 \pm 1.0$ & $1.6 \pm 0.7$ & $\mathrm{~ns}$ \\
Glucose [mmol/L] & $6.6 \pm 1.9$ & $5.6 \pm 0.8$ & $\mathrm{~ns}$ \\
Uric acid [mg/dL] & $4.9 \pm 1.2$ & $5.4 \pm 1.4$ & $\mathrm{~ns}$ \\
Fibrinogen [g/L] & $4.4 \pm 1.8$ & $4.1 \pm 1.1$ & $\mathrm{~ns}$ \\
Apo A-I [g/L] & $1.4 \pm 0.3$ & $1.5 \pm 0.2$ & $\mathrm{~ns}$ \\
Apo B [g/L] & $1.0 \pm 0.2$ & $1.1 \pm 0.2$ & $\mathrm{~ns}$ \\
Apo B/A-I & $0.8 \pm 0.2$ & $0.7 \pm 0.2$ & $\mathrm{~ns}$ \\
C-reactive protein [mg/L] & $12.3 \pm 13.7$ & $7.5 \pm 11.9$ & $\mathrm{~ns}$ \\
Body mass index & $25.5 \pm 4.3$ & $23.3 \pm 3.6$ & $\mathrm{~ns}$ \\
Age [years] & $61 \pm 8$ & $62 \pm 7$ & $\mathrm{~ns}$ \\
\hline
\end{tabular}

HDL: high-density lipoprotein; LDL: low-density lipoprotein; Apo A-I: apolipoprotein A-I; and Apo B: apolipoprotein B.

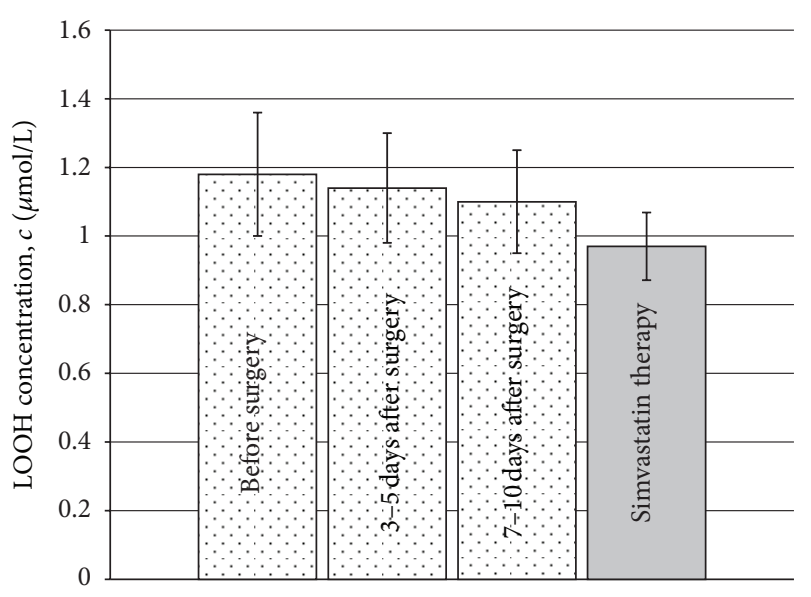

Figure 1: Concentrations of lipid hydroperoxides (LOOHs) in Group I patients before surgery, in the postoperative phase, and on simvastatin therapy.

and 7-10 days after surgery in Group I, in comparison to the values before and 3-5 days after surgery (Figure 5).

In addition, all the studied parameters $(\mathrm{LOOH}, \mathrm{MDA}$, FRAP, -SH groups, and ceruloplasmin) in Group I patients before surgery were significantly correlated with mean changes at 3-5 days $\left(\Delta_{1}\right)$ and/or 7-10 days $\left(\Delta_{2}\right)$ after surgery (Table 2).

\section{Discussion}

Oxidized LDL is a biochemical marker of oxidative damage and has atherogenic effects, including the stimulation of foam cell formation and activation of the inflammatory process in the vascular wall [29]. The findings of the present study are broadly consistent with the hypothesis that lipid peroxidation may be one mechanism through which several risk factors may promote PAD.

MDA and $\mathrm{LOOH}$ are suitable markers for evaluating oxidative stress. In our study, we observed an increased MDA

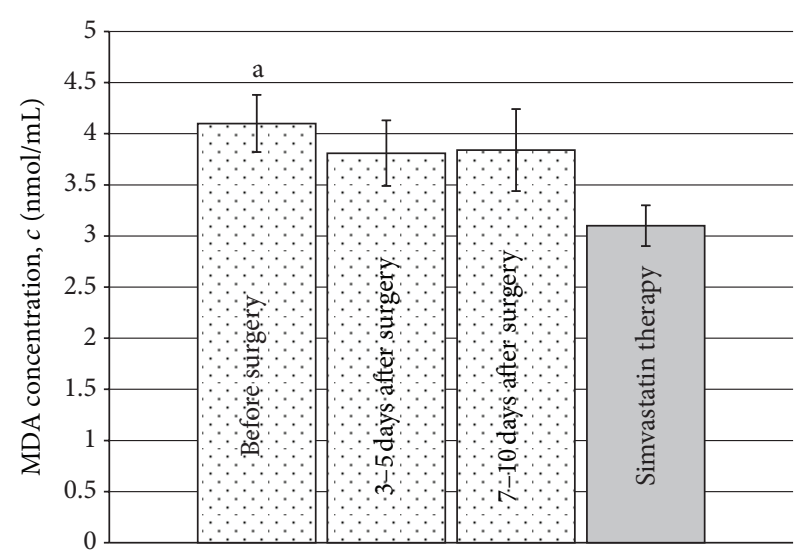

FIGURE 2: Malondialdehyde (MDA) concentrations in Group I patients before surgery, in the postoperative phase, and on simvastatin therapy. a-versus simvastatin therapy; $P<0.05$.

TABLE 2: Pearson's correlation coefficients $r_{1}$ and $r_{2}$ for the analyzed parameters before surgery versus $3-5$ days $\left(\Delta_{1}\right)$ and $7-10$ days $\left(\Delta_{2}\right)$ after surgery, respectively.

\begin{tabular}{lccc}
\hline Parameter & $r_{1}\left(\Delta_{1}\right)$ & $r_{2}\left(\Delta_{2}\right)$ & $P$ value \\
\hline MDA & -0.575 & -0.516 & $<0.05$ \\
LOOH & -0.671 & -0.778 & $<0.05$ \\
FRAP & -0.555 & -0.637 & $<0.05$ \\
Thiol groups & -0.664 & -0.599 & $<0.05$ \\
Ceruloplasmin & n.d. & -0.682 & $<0.05$ \\
\hline
\end{tabular}

MDA: malondialdehyde; LOOHs: lipid hydroperoxides; FRAP: ferric reducing antioxidant power; and n.d.: no difference.

level in the patients before surgery, which suggests that they were in a state of high oxidative stress. At neutral pH, MDA exists as a low reactive enolate anion, but it is still toxic and may damage many biologically important molecules. Determination of the lipid peroxidation product MDA in PAD is important because, as was shown earlier, MDA damages 


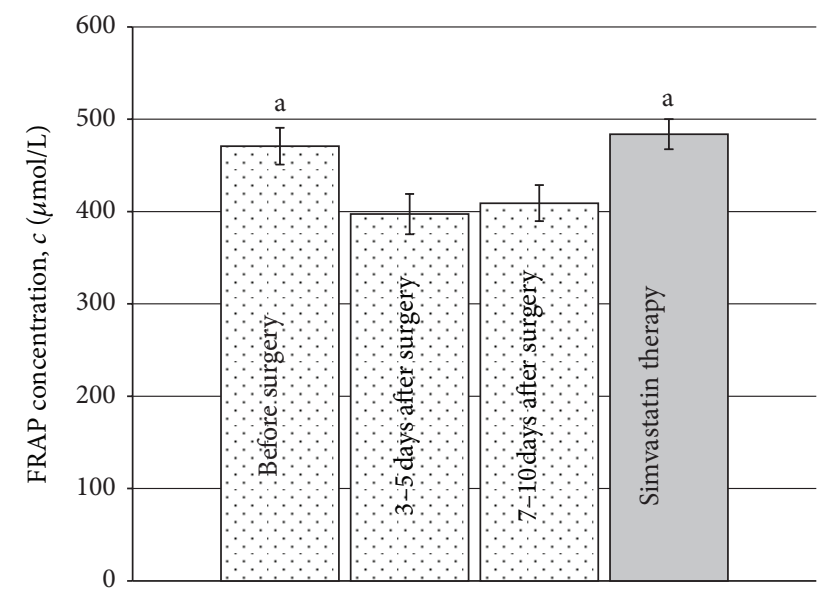

FIGURE 3: Ferric reducing antioxidant power (FRAP) in Group I patients before surgery, in the postoperative phase, and on simvastatin therapy. a-versus 3-5 days and 7-10 days after surgery; $P<0.05$.

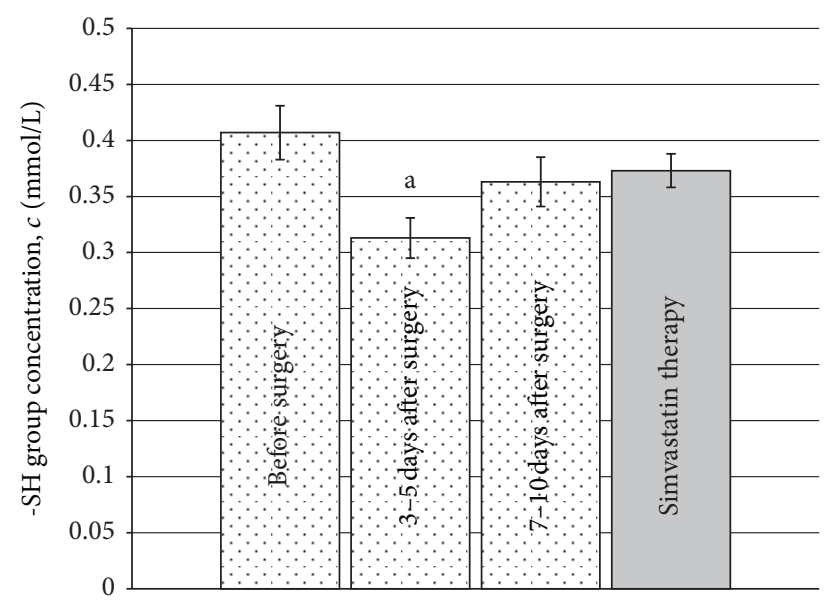

FIgURE 4: Thiol (-SH) group concentrations in Group I patients before surgery, in the postoperative phase, and on simvastatin therapy. a-versus before surgery, 7-10 days after surgery, and simvastatin therapy; $P<0.05$.

collagen by forming transverse intramolecular bonds that cause rigidity of blood vessels [30]. In addition, modification of native LDL by MDA causes the accumulation of cholesteryl esters within the cell in which the atherosclerotic reaction takes place [31].

Several trials have shown that statin therapy relieves symptoms and improves function in patients with lower extremity PAD [32,33]. MDA concentrations in the serum of the patients treated with simvastatin were lower than those in Group I patients before surgery. Since PAD patients are usually undertreated with regard to the use of lipid-lowering agents, compared to patients with coronary heart disease, our results provide strong support for the efficacy of statin therapy in PAD.

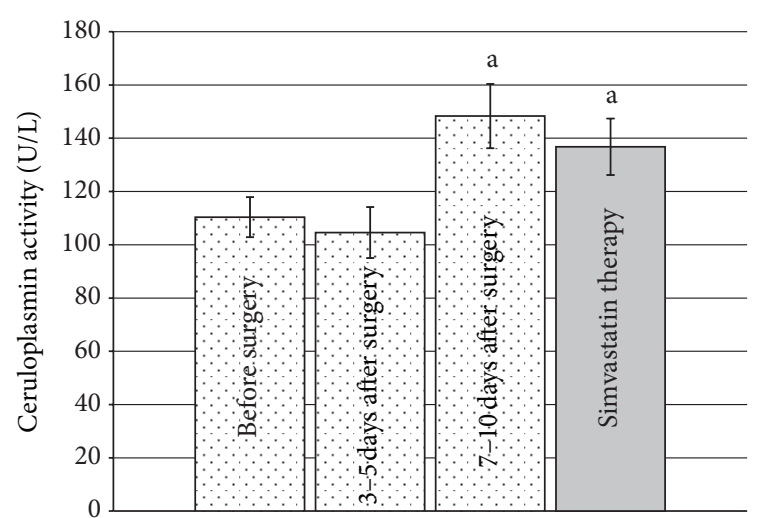

FIGURE 5: Ceruloplasmin activity in Group I patients before surgery, in the postoperative phase, and on simvastatin therapy. a-versus before surgery and 3-5 days after surgery; $P<0.05$.

Human cells and tissues contain many antioxidant enzyme systems and nonenzymatic antioxidants, which protect against the action of toxic free radicals [34]. The FRAP assay used here is a useful indicator of the body's antioxidant status, which determines the level of non-enzymatic antioxidants, such as uric acid, ascorbic acid, $\alpha$-tocopherol, and some protein-containing -SH groups.

An increase in FRAP level in the patients before surgical treatment is consistent with similar results obtained by Gawron et al. [35] in a group of patients with coronary heart disease and by Lantos et al. [36] in patients with hypertension. An increased antioxidant capacity of serum seems to be response to an increase in the concentration of lipid peroxidation products.

The reduction in FRAP level observed in our Group I patients 3-5 and 7-10 days after surgery can be partly explained by a decrease in their serum antioxidant status and an increase in serum oxidant levels following surgery, as indicated by the negative correlations found (Table 2). In their study, Girona et al. [37] analyzed the antioxidant effect of simvastatin both in vitro and in vivo. They showed that simvastatin therapy increases the resistance of both LDL and HDL to oxidation. In addition, simvastatin inhibits the ability of activated macrophages to oxidize LDL in vitro in a dosedependent manner [38]. A similar effect has been shown for other statins, such as atorvastatin [39], which reduces LDL levels and HDL oxidation and protects paraoxonasean enzyme bound to HDL.

With regard to FRAP in our study, simvastatin therapy (20 mg daily) did not influence the total antioxidant potential. However, Shin et al. [40] found a significant increase in antioxidant ability in their group of hypercholesterolemic patients treated with $40 \mathrm{mg}$ simvastatin daily. They suggested the possibility of a dose-dependent effect of simvastatin on plasma antioxidant status. This could be the reason why we did not detect any effect of simvastatin on FRAP in our study.

Glutathione and other proteins, such as albumin, are an important part of serum antioxidant activity against free radicals. Wayner et al. [41] believe that protein participation makes up to $50 \%$ of the antioxidant status. The thiol groups 
react with peroxide radicals in the first stage of the oxidation reaction and also act in the lag phase, which is an accepted marker of serum oxidizability. In our group of patients with $\mathrm{PAD}$, the decrease in thiol group concentrations, observed 35 days after surgery, followed by an increase 7-10 days after surgery, may be explained by oxidative stress intensification in reperfusion and by the actions of - $\mathrm{SH}$ groups, leading to their reduction. Furthermore, a decrease in both lag phase and albumin level has also been reported in the serum of patients with aneurysmal or arterial occlusive disease [42].

Some studies have confirmed that ceruloplasmin is an independent risk factor for cardiovascular disease [43, 44]. Elevated serum ceruloplasmin activity has been reported in patients with atherosclerosis obliterans [45]. Authors of that study suggest that increased ceruloplasmin activity is related to the acute phase response to inflammation and necrosis in atherosclerosis obliterans and results from increased ROS production, mostly of superoxide radicals. Ceruloplasmin contains four free thiol groups [46]. Therefore, the preferential oxidative destruction of thiol groups can explain the greater susceptibility of ceruloplasmin to oxidative stress.

Recent data show that statins may reduce oxidative stress [47] by decreasing the generation of ROS, thereby synergizing with the biological effects of antioxidants [48]. The results of our study show that ceruloplasmin activity was influenced by both simvastatin therapy and surgical treatment, but only 7-10 days postoperatively. In our opinion, simvastatin may be implicated in antioxidant properties of ceruloplasmin, as reflected by the increase in its activity in men on simvastatin therapy. Several in vitro studies have indicated that ceruloplasmin is a potent catalyst of LDL oxidation and that has been attributed to the presence of a reduced copper atom on its surface. ROS dissociate copper from ceruloplasmin and therefore can markedly alter its structure and function, which could explain the initial lack of differences in its activity 3-5 days after surgery.

In view of the escalating prevalence of PAD in our aging population, further studies are needed to clarify precisely not only the mechanisms involved but the relative importance of the lipid-related and nonlipid-related effects of statins as well.

\section{Conclusions}

Results of this study show diverse oxidative state responses to surgical treatment and confirm the beneficial effects of statin therapy in PAD.

\section{Conflict of Interests}

The authors declare that there is no conflict of interests regarding the publication of this paper.

\section{References}

[1] G. J. Hankey, P. E. Norman, and J. W. Eikelboom, "Medical treatment of peripheral arterial disease," The Journal of the American Medical Association, vol. 295, no. 5, pp. 547-553, 2006.

[2] B. A. Golomb, T. T. Dang, and M. H. Criqui, "Peripheral arterial disease: morbidity and mortality implications," Circulation, vol. 114, no. 7, pp. 688-699, 2006.
[3] A. B. Newman, L. Shemanski, T. A. Manolio et al., "Ankle-arm index as a predictor of cardiovascular disease and mortality in the Cardiovascular Health Study," Arteriosclerosis, Thrombosis, and Vascular Biology, vol. 19, no. 3, pp. 538-545, 1999.

[4] E. P. Brass, L. T. Cooper, P. Hanson, and W. R. Hiatt, "Association of clinical attributes and treadmill walking performance in patients with claudication due to peripheral artery disease," Journal of Vascular Surgery, vol. 58, no. 2, pp. 396-403, 2013.

[5] A. T. Hirsch, M. H. Criqui, D. Treat-Jacobson et al., "Peripheral arterial disease detection, awareness, and treatment in primary care," The Journal of the American Medical Association, vol. 286, no. 11, pp. 1317-1324, 2001.

[6] A. V. Meru, S. Mittra, B. Thyagarajan, and A. Chugh, "Intermittent claudication: an overview," Atherosclerosis, vol. 187, no. 2, pp. 221-237, 2006.

[7] M. H. Criqui, R. D. Langer, A. Fronek et al., "Mortality over a period of 10 years in patients with peripheral arterial disease," The New England Journal of Medicine, vol. 326, no. 6, pp. 381386, 1992.

[8] A. B. Newman, K. Sutton-Tyrrell, M. T. Vogt, and L. H. Kuller, "Morbidity and mortality in hypertensive adults with a low ankle/arm blood pressure index," The Journal of the American Medical Association, vol. 270, no. 4, pp. 487-489, 1993.

[9] F. J. Khawaja and I. J. Kullo, "Novel markers of peripheral arterial disease," Vascular Medicine, vol. 14, no. 4, pp. 381-392, 2009.

[10] M. R. Banta, F. Ma, D. M. Bravata, R. S. Kirsner, and D. G. Federman, "Incidence of and factors associated with achieving target lipid levels in patients with peripheral arterial disease," Journal of General Internal Medicine, vol. 21, no. 7, pp. 711-714, 2006.

[11] P. W. F. Wilson, R. B. D’Agostino, D. Levy, A. M. Belanger, H. Silbershatz, and W. B. Kannel, "Prediction of coronary heart disease using risk factor categories," Circulation, vol. 97, no. 18, pp. 1837-1847, 1998.

[12] M. Rizzo, G. Montalto, and M. Banach, "The effects of statins on blood pressure: current knowledge and future perspectives," Archives of Medical Science, vol. 8, no. 1, pp. 1-3, 2012.

[13] A. S. Wierzbicki, R. Poston, and A. Ferro, "The lipid and nonlipid effects of statins," Pharmacology and Therapeutics, vol. 99, no. 1, pp. 95-112, 2003.

[14] M. Pioruńska-Stolzmann and A. Pioruńska-Mikołajczak, "The influence of simvastatin on lipase and cholesterol esterase activity in the serum of men with coronary heart disease," Pharmacological Research, vol. 43, pp. 359-362, 2001.

[15] G. Gargiulo, G. Giugliano, L. Brevetti et al., "Use of statins in lower extremity artery disease: a review," BMC Surgery, vol. 12, supplement 1, p. S15, 2012.

[16] G. Erez and E. Leitersdorf, "The rationale for using HMG-CoA reductase inhibitors ('statins') in peripheral arterial disease," European Journal of Vascular and Endovascular Surgery, vol. 33, no. 2, pp. 192-201, 2007.

[17] R. S. Rosenson, "Statins in atherosclerosis: lipid-lowering agents with antioxidant capabilities," Atherosclerosis, vol. 173, no. 1, pp. $1-12,2004$.

[18] R. Di Mascio, R. Marchioli, and G. Tognoni, "Cholesterol reduction and stroke occurrence: an overview of randomized clinical trials," Cerebrovascular Diseases, vol. 10, no. 2, pp. 85-92, 2000.

[19] R. P. Byington, B. R. Davis, J. F. Plehn et al., "Reduction of stroke events with pravastatin: the Prospective Pravastin Pooling (PPP) Project," Circulation, vol. 103, no. 3, pp. 387-392, 2001. 
[20] T. B. Horwich, W. R. MacLellan, and G. C. Fonarow, "Statin therapy is associated with improved survival in ischemic and non-ischemic heart failure," Journal of the American College of Cardiology, vol. 43, no. 4, pp. 642-648, 2004.

[21] A. Hognestad, K. Dickstein, E. Myhre, S. Snapinn, and J. Kjekshus, "Effect of combined statin and beta-blocker treatment on one-year morbidity and mortality after acute myocardial infarction associated with heart failure," American Journal of Cardiology, vol. 93, no. 5, pp. 603-606, 2004.

[22] K. I. Paraskevas, A. S. Wierzbicki, and D. P. Mikhailidis, "Statins and noncardiac vascular disease," Current Opinion in Cardiology, vol. 27, no. 4, pp. 392-397, 2012.

[23] R. L. Pande, T. S. Perlstein, J. A. Beckman, and M. A. Creager, "Secondary prevention and mortality in peripheral artery disease: national health and nutrition examination study, 1999 to 2004," Circulation, vol. 124, no. 1, pp. 17-23, 2011.

[24] I. F. F. Benzie and J. J. Strain, "Ferric reducing/antioxidant power assay: direct measure of total antioxidant activity of biological fluids and modified version for simultaneous measurement of total antioxidant power and ascorbic acid concentration," Methods in Enzymology, vol. 299, pp. 15-27, 1999.

[25] E. Södergren, J. Nourooz-Zadeh, L. Berglund, and B. Vessby, "Re-evaluation of the ferrous oxidation in xylenol orange assay for the measurement of plasma lipid hydroperoxides," Journal of Biochemical and Biophysical Methods, vol. 37, no. 3, pp. 137-146, 1998.

[26] H. Ohkawa, N. Ohishi, and K. Yagi, "Assay for lipid peroxides in animal tissues by thiobarbituric acid reaction," Analytical Biochemistry, vol. 95, no. 2, pp. 351-358, 1979.

[27] M.-L. Hu, "Measurement of protein thiol groups and glutathione in plasma," Methods in Enzymology, vol. 233, pp. 380$385,1994$.

[28] K. H. Schosinsky, H. P. Lehmann, and M. F. Beeler, "Measurement of ceruloplasmin from its oxidase activity in serum by use of o dianisidine dihydrochloride," Clinical Chemistry, vol. 20, no. 12, pp. 1556-1563, 1974.

[29] G. K. Hansson, A. K. Robertson, and C. Söderberg-Nauclér, "Inflammation and atherosclerosis," Annual Review of Pathology, vol. 1, pp. 297-329, 2006.

[30] D. del Rio, A. J. Stewart, and N. Pellegrini, "A review of recent studies on malondialdehyde as toxic molecule and biological marker of oxidative stress," Nutrition, Metabolism and Cardiovascular Diseases, vol. 15, no. 4, pp. 316-328, 2005.

[31] A. M. Fogelman, I. Shechter, J. Seager, M. Hokom, J. S. Child, and P. A. Edwards, "Malondialdehyde alteration of low density lipoproteins leads to cholesteryl ester accumulation in human monocyte-macrophages," Proceedings of the National Academy of Sciences of the United States of America, vol. 77, no. 4, pp. 22142218, 1980.

[32] J. Giri, M. M. McDermott, P. Greenland et al., "Statin use and functional decline in patients with and without peripheral arterial disease," Journal of the American College of Cardiology, vol. 47, no. 5, pp. 998-1004, 2006.

[33] D. G. Hackam, N. M. Sultan, and M. H. Criqui, "Vascular protection in peripheral artery disease: systematic review and modelling study," Heart, vol. 95, no. 13, pp. 1098-1102, 2009.

[34] H. Sies, "Strategies of antioxidant defense," European Journal of Biochemistry, vol. 215, no. 2, pp. 213-219, 1993.

[35] A. Gawron, J. Chrzczanowicz, D. Nowak et al., "Total antioxidant capacity of blood plasma in healthy men and in men with coronary heart disease," Przeglad Lekarski, vol. 62, no. 3, pp. 3134, 2005.
[36] J. Lantos, E. Röth, L. Czopf, J. Nemes, and I. Gál, “Monitoring of plasma total antioxidant status in different diseases," Acta Chirurgica Hungarica, vol. 36, no. 1-4, pp. 188-189, 1997.

[37] J. Girona, A. E. la Ville, R. Solà, N. Plana, and L. Masana, "Simvastatin decreases aldehyde production derived from lipoprotein oxidation," American Journal of Cardiology, vol. 83, no. 6, pp. 846-851, 1999.

[38] L. M. Giroux, J. Davignon, and M. Naruszewicz, "Simvastatin inhibits the oxidation of low-density lipoproteins by activated human monocyte-derived macrophages," Biochimica et Biophysica Acta, vol. 1165, no. 3, pp. 335-338, 1993.

[39] M. Aviram, M. Rosenblat, C. L. Bisgaier, and R. S. Newton, "Atorvastatin and gemfibrozil metabolites, but not the parent drugs, are potent antioxidants against lipoprotein oxidation," Atherosclerosis, vol. 138, no. 2, pp. 271-280, 1998.

[40] M.-J. Shin, N. Chung, J. H. Lee et al., "Effects of simvastatin on plasma antioxidant status and vitamins in hypercholesterolemic patients," International Journal of Cardiology, vol. 118, no. 2, pp. 173-177, 2007.

[41] D. D. M. Wayner, G. W. Burton, K. U. Ingold, L. R. C. Barclay, and S. J. Locke, "The relative contributions of vitamin E, urate, ascorbate and proteins to the total peroxyl radical-trapping antioxidant activity of human blood plasma," Biochimica et Biophysica Acta, vol. 924, no. 3, pp. 408-419, 1987.

[42] I. Delimaris, S. Georgopoulos, C. Kroupis et al., "Serum oxidizability, total antioxidant status and albumin serum levels in patients with aneurysmal or arterial occlusive disease," Clinical Biochemistry, vol. 41, no. 9, pp. 706-711, 2008.

[43] R. L. Atanasiu, D. Stea, M. A. Mateescu et al., "Direct evidence of caeruloplasmin antioxidant properties," Molecular and Cellular Biochemistry, vol. 189, no. 1-2, pp. 127-135, 1998.

[44] P. L. Fox, C. Mukhopadhyay, and E. Ehrenwald, "Structure, oxidant activity, and cardiovascular mechanisms of human ceruloplasmin," Life Sciences, vol. 56, no. 21, pp. 1749-1758, 1995.

[45] M. Iskra and W. Majewski, "Oxidase activity of ceruloplasmin and concentrations of copper and zinc in serum in chronic arterial occlusion of the lower limbs," Journal of Trace Elements in Medicine and Biology, vol. 13, no. 1-2, pp. 76-81, 1999.

[46] N. Shukla, J. Maher, J. Masters, G. D. Angelini, and J. Y. Jeremy, "Does oxidative stress change ceruloplasmin from a protective to a vasculopathic factor?" Atherosclerosis, vol. 187, no. 2, pp. 238-250, 2006.

[47] E. M. Aguilar, J. de Haro Miralles, A. F. González, C. V. Casariego, S. B. Moreno, and F. A. García, "In vivo confirmation of the role of statins in reducing nitric oxide and C-reactive protein levels in peripheral arterial disease," European Journal of Vascular and Endovascular Surgery, vol. 37, no. 4, pp. 443-447, 2009.

[48] E. C. Pereira, M. C. Bertolami, A. A. Faludi, A. Sevanian, and D. S. P. Abdalla, "Antioxidant effect of simvastatin is not enhanced by its association with $\alpha$-tocopherol in hypercholesterolemic patients," Free Radical Biology and Medicine, vol. 37, no. 9, pp. 1440-1448, 2004. 


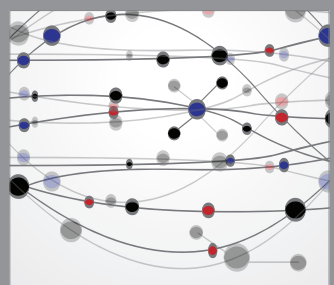

The Scientific World Journal
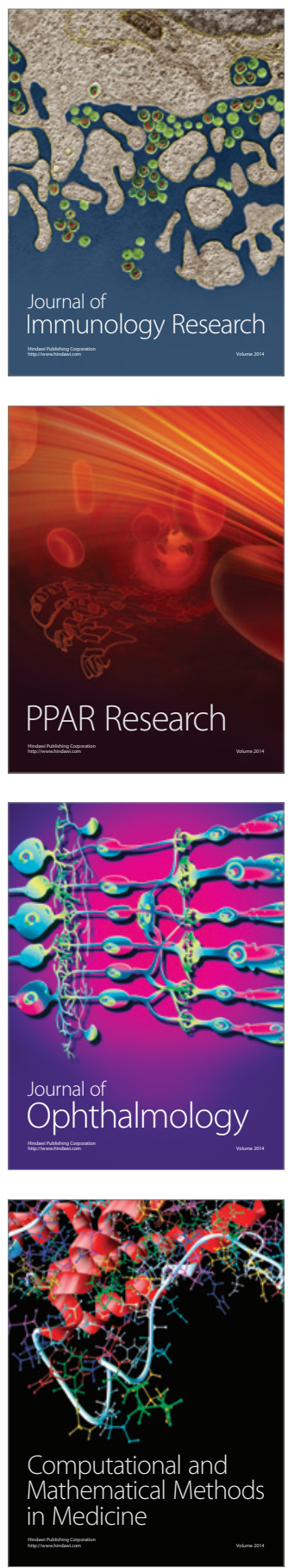

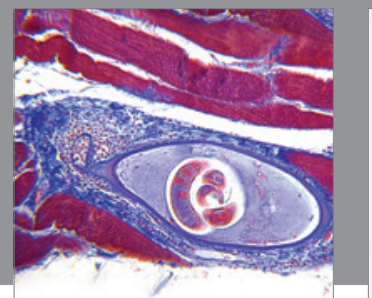

Gastroenterology

Research and Practice
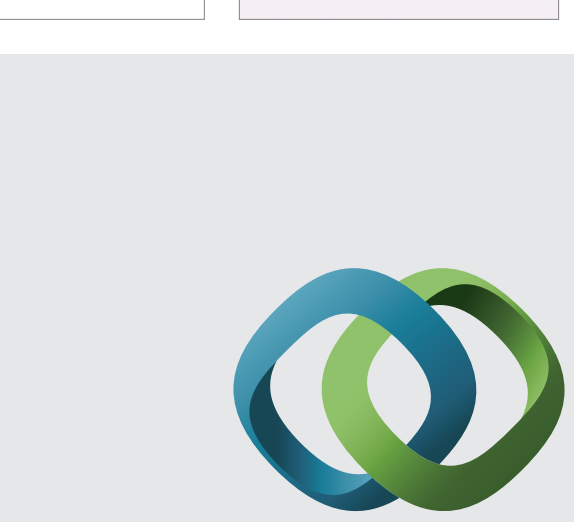

\section{Hindawi}

Submit your manuscripts at

http://www.hindawi.com
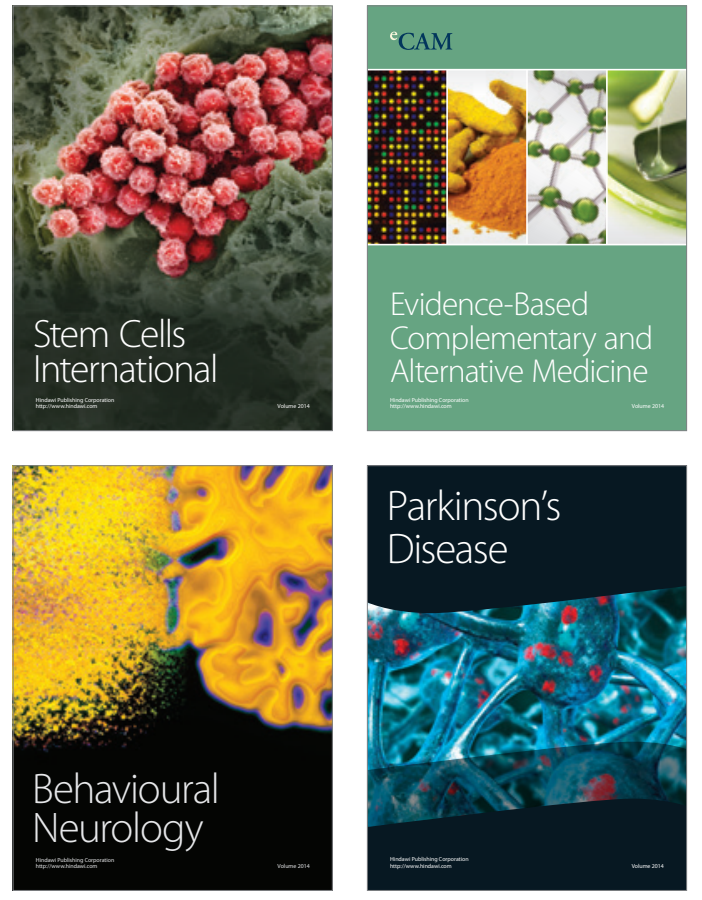
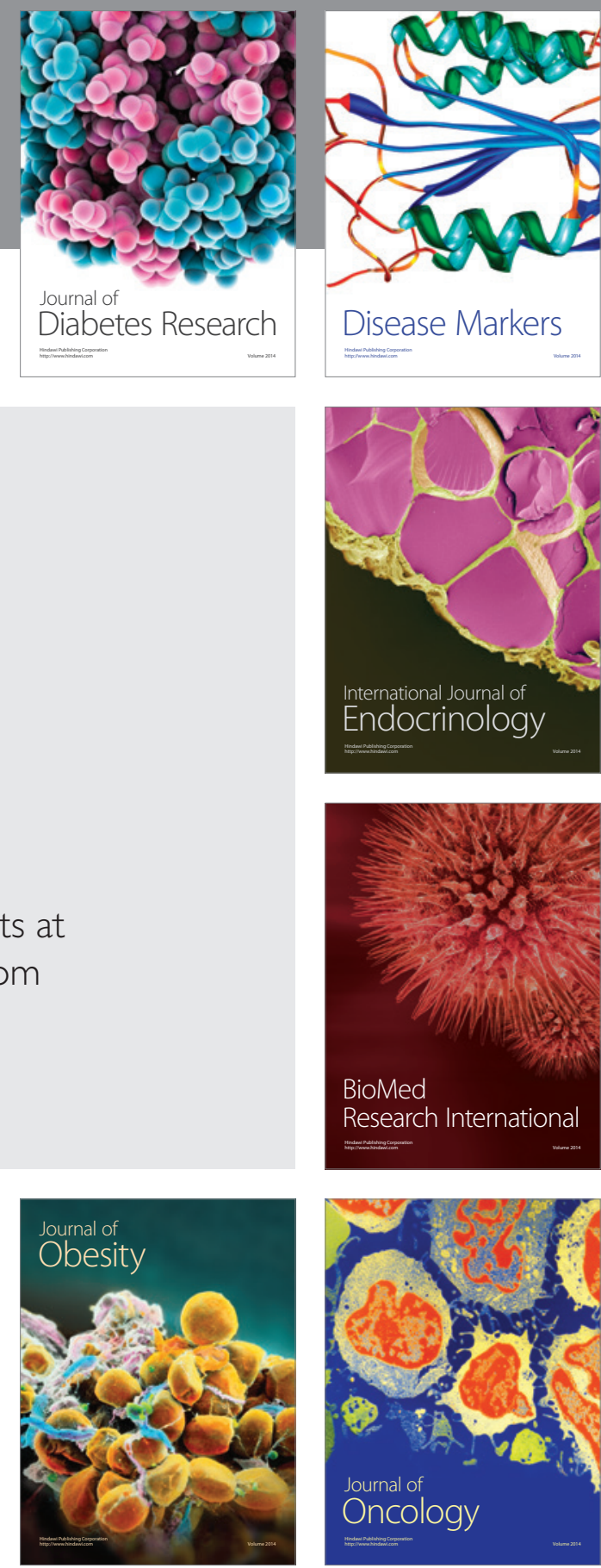

Disease Markers
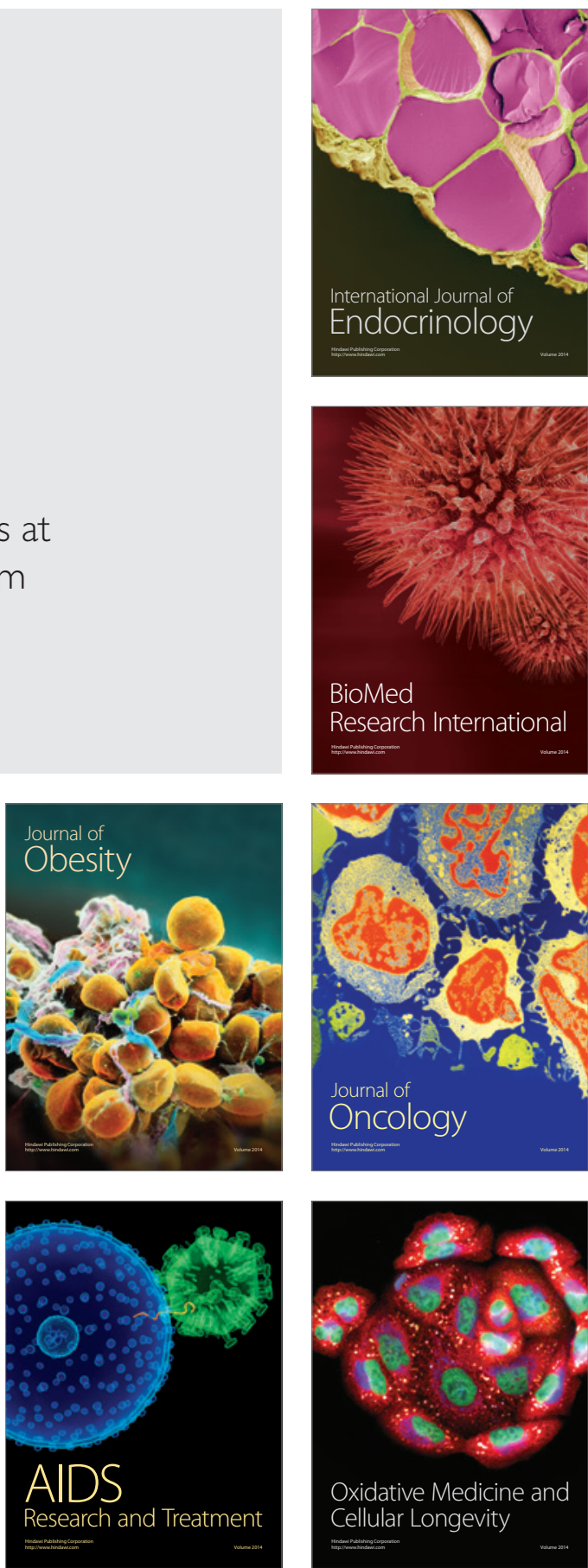